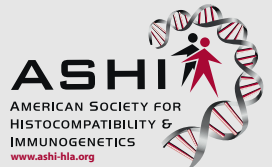

journal homepage: www.elsevier.com/locate/humimm

\title{
Mycobacterium tuberculosis may escape helper T cell recognition by infecting human fibroblasts
}

\author{
Sabrina Mariotti ${ }^{a}$, Valeria Sargentini ${ }^{a}$, Manuela Pardini ${ }^{a}$, Federico Giannoni ${ }^{a}$, Marco De Spirito ${ }^{b}$, \\ Maria Cristina Gagliardi ${ }^{a}$, Emanuela Greco ${ }^{c}$, Raffaela Teloni ${ }^{a}$, Maurizio Fraziano ${ }^{c}$, Roberto Nisini ${ }^{\mathrm{a}, *}$ \\ ${ }^{a}$ Dipartimento di Malattie Infettive, Parassitarie e Immunomediate, Istituto Superiore di Sanità, Roma, Italy \\ ' Istituto di Fisica, Universita' Cattolica del Sacro Cuore, Roma, Italy \\ 'Dipartimento di Biologia, Universita' di Roma “Tor Vergata”, Roma, Italy
}

\section{A R T I C L E I N F O}

\section{Article history:}

Received 13 June 2012

Accepted 19 February 2013

Available online $\mathrm{xxxx}$

\begin{abstract}
A B S T R A C T
The host immune response can limit Mycobacterium tuberculosis (Mtb) spreading in primary tuberculosis (TB) without eradicating all bacilli, which can persist causing latent TB infection and are responsible for reactivation TB. Persistent Mtb is confined to granulomas within phagocytes, but it is also found in other non-immune cells. We focused on fibroblasts since these cells participate to the granuloma formation and were shown to be infected in latent TB infections.

We show that in vitro both Mtb and Bacille Calmette-Guérin actively replicate in human fibroblasts. Mycobacterial infection of fibroblasts causes a significant inhibition of interferon (IFN)- $\gamma$ induced membrane expression of major histocompatibility complex class II molecules in these cells. The functional consequence of in vitro infection is a significant reduction of the fibroblast capacity to present peptides and soluble proteins to autologous specific $\mathrm{CD} 4^{+} \mathrm{T}$ cell clones. Moreover, fibroblasts are capable of presenting antigen derived from the processing of heat-killed Mtb, but not from viable Mtb. Data indicate that IFN- $\gamma$ treated fibroblasts are capable of presenting antigens derived from the processing of whole bacteria in addition to the capacity to present peptides and isolated proteins. Interestingly, Mtb infected fibroblasts lose this capacity, suggesting that Mtb may evade T helper immune surveillance by infecting fibroblasts.

(c) 2013 American Society for Histocompatibility and Immunogenetics. Published by Elsevier Inc. All rights
\end{abstract} reserved.

\section{Introduction}

The intracellular pathogen Mycobacterium tuberculosis (Mtb) causes tuberculosis (TB) and is able to infect antigen presenting cells (APCs), including macrophages and dendritic cells (DCs) [1]. In these cells, Mtb survives in modified phagosomes and uses multiple mechanisms to evade both innate and adaptive host immunity, including inhibition of phagosome maturation, resistance to innate microbicidal mechanisms and cytokine-mediated host defenses, as well as inhibition of antigen presentation [2,3]. Since

Abbreviations: APCs, antigen presenting cells; BCG, Bacillus Calmette-Guérin; CIITA, class II transcriptional transactivator; CFU, colony forming unit; CM, complete medium; DCs, dendritic cells; FCS, fetal calf serum; IFN, interferon; LTBI, latent TB infection; MHC, major histocompatibility complex; MOI, multiplicity of infection; Mtb, Mycobacterium tuberculosis; RNFib, primary autologous fibroblasts; $\mathrm{PPD}$, purified protein derivative; TB, tuberculosis.

* Corresponding author. Address: Dipartimento di Malattie Infettive, Parassitarie e Immunomediate, Istituto Superiore di Sanita', Viale Regina Elena 299, 00161 Roma, Italy. Fax: + 390649387112.

E-mail address: roberto.nisini@iss.it (R. Nisini).
TB is mainly transmitted via airborne droplets from people with active respiratory disease to susceptible individuals, Mtb most commonly affects the lungs. Thus, it is generally accepted that the first cell types confronting Mtb during primary infection are alveolar macrophages and type II pneumocytes $[4,5]$. However, this microorganism can enter a variety of other cell types in vitro, as shown by several authors following the early observations by Shepard, [6] who demonstrated that Mtb can enter monolayers of HeLa, monkey kidney and human amnion cells [6,7]. More recently, Mtb has been shown to infect lung epithelial and endothelial cells [8-11] as well as adipocytes [12] and fibroblasts [13,14], in addition to professional APCs, such as monocytes and DCs, even if the capacity of Mtb to replicate within these different cell types may vary significantly $[15,16]$. The capacity of Mtb to infect cells different from macrophages has also been proven by ex vivo experiments showing Mtb or its DNA in different cell types in both TB patients and subjects with latent TB infection (LTBI), corroborating the significance of in vitro experiments $[12,17]$. In particular, Mtb was shown to persist intracellularly in lung tissue without histological evidence of tuberculous lesions and Mtb DNA was shown 
to be situated not only in macrophages but also in other non-professional APCs [17]. The capacity of Mtb to infect and replicate within different cell types may be dependent on both the capacity of cells to internalize and host Mtb and/or to the strength of the immune response, which may force Mtb to colonize alternative cell types possibly in relation to the TB stage. This is relevant since internalization in non-professional APCs may represent an important pathogenic feature of mycobacterial infection. In these cells major histocompatibility complex (MHC) class I expression is constitutive but class II is not expressed in normal conditions, although it may be induced by interferon (IFN)- $\gamma$ release [18]. Non-professional APCs are not capable of priming antigen specific $\mathrm{T}$ cells, but they can present antigen to memory $\mathrm{CD} 4^{+} \mathrm{T}$ cells in inflammatory microenvironments where they are induced to express MHC class II molecules by locally released IFN- $\gamma$. The capacity of mycobacteria to infect, survive and interfere with antigen processing and presentation in these cells is far from being elucidated [19]. Moreover the role of non-professional APCs in TB is intriguing in the light of their possible contribution in maintaining the infection latent and possibly representing the mycobacterial reservoir in LTBI [17]. In this paper we investigated whether the known capacity of Mtb to interfere with MHC class II expression and the consequent impairment of antigen presentation could be observed in non professional APCs such as fibroblasts. In fact, fibroblasts are recruited [20] and proliferate into TB lesions where they are involved in tissue remodeling and granuloma formation, thus representing possible target for Mtb infection.

\section{Materials and methods}

\subsection{Ethics statement}

This study has been approved by the Istituto Superiore di Sanità review board and written informed consent has been obtained by the healthy volunteers who participated to the study.

\subsection{Bacterial cultures}

Mtb H37Rv (ATCC 27294) and Bacillus Calmette-Guérin (BCG), (ATCC 27291) were grown with gentle agitation (80 r.p.m.) in Middlebrook 7H9 broth (Difco Laboratories, Detroit, MI) supplemented with $0.05 \%$ Tween 80 (Sigma Chemical Company, St. Louis, MO) and 10\% Middlebrook ADC enrichment (Becton Dickinson, Mountain View, CA) as previously described [21]. Logarithmically growing cultures were washed two times in RPMI 1640. Mycobacteria were resuspended in RPMI 1640 containing 10\% heat inactivated fetal calf serum (FCS) and then stored at $-80{ }^{\circ} \mathrm{C}$. Vials were thawed, and bacterial viability was determined by counting the number of colony forming unit (CFU) on Middlebrook 7H10 agar plates. For some experiments, Mtb was heat killed at $80^{\circ} \mathrm{C}$ for one hour (HK Mtb).

All Mtb and BCG preparations were analyzed for LPS contamination by the Limulus lysate assay (BioWhittaker) and contained less than $10 \mathrm{pg} / \mathrm{ml}$ of LPS.

\subsection{Fibroblasts}

MRC-5 cells (human fetal lung fibroblasts; European Collection of Cell Cultures (ECACC no. 84101801) were cultured in RPMI 1640, supplemented with $1 \mathrm{mM}$ glutamine, $1 \mathrm{mM}$ sodium pyruvate, $1 \%$ nonessential amino acids, and 10\% FCS (complete medium, CM) (all from Euroclone Ltd., UK) in tissue culture flasks (Becton Dickinson, France) maintained at $37^{\circ} \mathrm{C}$ in $5 \% \mathrm{CO}_{2}$. From the same donor (RN) of antigen specific T cell clones (see below), who gave his informed consent to participate to the study, a skin biopsy was ob- tained and primary autologous fibroblasts (RNFib) were derived as previously described [22]. Briefly, 3-mm skin punch biopsies were cut into small fragments (approx. 1-mm) and cultured in RPMI 1640 medium containing 20\% FCS. After the skin fibroblasts had grown out of the explants and reached confluence, they were trypsinized and transferred to tissue culture flasks. These cells, RNFib, were maintained in culture in CM and split every 1-2 weeks as they reached confluence. All experiments were performed with skin fibroblasts taken after 10 to 20 culture passages.

\subsection{Fibroblasts infection}

MRC-5 and primary RNFib fibroblasts were harvested from tissue culture flasks by trypsin-EDTA (Euroclone, Italy). After washings, fibroblasts were counted and plated at $1 \times 10^{5}$ cell/well into 24-well plates. Cells were allowed to adhere and then cells were infected with Mtb or BCG at multiplicity of infection (MOI) of 3:1 and 10:1 respectively for 18 hours. Then wells were washed several times using warm RPMI and eventually fresh CM was added to the wells (T0). To measure mycobacterial growth, after $24 \mathrm{~h}$, $72 \mathrm{~h}$ and 6 days of culture supernatants were collected and the cells lysed using distilled water containing $0.1 \%$ saponin for $5 \mathrm{~min}$ at room temperature. Then, CFU for each well were separately calculated in supernatants and cell lysates, with the exception of the well representative of $T 0$, where only intracellular CFU were calculated. For CFU count, cell lysates and supernatants were briefly sonicated and 10 -fold dilutions were performed using $0.05 \%$ tween 80 in distilled water and serial dilutions were plated on Middlebrook $7 \mathrm{H} 10$. After 21 days of incubation at $37^{\circ} \mathrm{C}$ in $5 \%$ $\mathrm{CO}_{2}$ atmosphere, $\mathrm{CFU}$ in each plate were enumerated. Values are expressed as a mean of three independent experiments \pm standard deviation.

\subsection{RNA extraction and $q R T-P C R$}

Human fibroblasts were lysed in TRIzol Reagent (Invitrogen Carlsbad, CA, USA), total RNA purified with SV Total RNA Isolation System (Promega, Madison, WI, USA), DNase I treated (Promega) and reverse transcribed using ImProm-II RT (Promega), according to manufacturer's instructions. qPCR was performed in an iCycler iQ (Bio-Rad, Hercules, Calif.) with iQ SYBR Green Supermix (BioRad) and $500 \mathrm{nM}$ each primer for 40 cycles as follows: $40 \mathrm{~s}$ at $95^{\circ} \mathrm{C}, 40 \mathrm{~s}$ at $57^{\circ} \mathrm{C}$, and $50 \mathrm{~s}$ at $72{ }^{\circ} \mathrm{C}$ for 40 cycles with a final melting curve. Beta-actin mRNA was used as housekeeping gene for $\Delta C t$ determination. Forward and reverse primers used for Beta-actin, $H L A-A, B$ and $C$, CIITA and HLA-DR $\alpha$ genes were: hBAct-S, 5'TCCTTCCTGGGCATGGAGTC-3', hBAct-AS, 5'-CAGGAGGAGCAATGA TCTTGATC-3', HLA-ABC-S, 5'-CTACGACGGCAAGGATTAC-3', HLAABC-AS, 5'-TTCCCGTTCTCCAGGTATC-3', CIITA-S, 5'-GGCTGGGAT TCCTACACAATG-3', CIITA-AS, 5'-CAACTCCATGGTGGCACAC-3' [23], HLA-DR $\alpha 3-S, 5^{\prime}$-CCCTGGGCCTGACTGTGG-3' and HLA-DR $\alpha 3-A S$, 5 -CCATCACCTCCATGTGCCTTAC-3'. The fold change compared to control untreated fibroblasts was calculated using the $2^{-\Delta \Delta C t}$ formula. All reactions were performed in triplicate.

\subsection{Microscopy}

Mtb and BCG infection of human fibroblasts was assessed using fibroblasts grown on cover-slip and stained with the Kinyoun method [21]. Intracellular localization was assessed by staining mycobacteria with auramine (Becton Dickinson, MD) [24] and analysis by confocal microscopy. Briefly, MRC-5 cell monolayer was exposed for $3 \mathrm{~h}$ to Mtb at the MOI of $1: 1$. Fibroblasts were washed with PBS to remove non-endocytosed bacilli, fixed with $4 \%$ paraformaldehyde for $10 \mathrm{~min}$ at $4{ }^{\circ} \mathrm{C}$ and finally washed with PBS. The localization of Mtb was determined by incubating in- 
fected monolayer with auramine for $20 \mathrm{~min}$ at room temperature, followed by 3 min incubation in $0.5 \%$ acid alcohol and repeated washings with PBS. Finally, cover slips were mounted using Vectashield mounting medium $\mathrm{H}-1000$ (Vector laboratories, CA) and their edges were sealed with nail polish. The samples were analyzed by confocal laser scanning microscopy using a Leica TCSSP2 operating system. Auramine was excited by using the $458 \mathrm{~nm}$ line of an argon laser for excitation, and fluorescence emission was collected from 500 to $530 \mathrm{~nm}$. Before each experiment, detector gain and laser intensity were adjusted to minimize background signals and held constant during acquisition.

\subsection{Flow cytometry}

Fibroblasts were infected as above described with BCG (MOI 10:1) and Mtb (MOI 3:1) for $18 \mathrm{~h}$. Fibroblast monolayer was then accurately washed in order to remove all the non-endocytosed/ bounded bacteria and stimulated for other $72 \mathrm{~h}$ with scalar doses of recombinant human IFN- $\gamma$ (12.5-400 ng/ml, Pharmingen; San Diego, CA, USA). To detect cell surface expression of HLA-A, B, C and HLA-DR, IFN- $\gamma$-stimulated and non infected, BCG or Mtb-infected cells were removed from wells with trypsin, washed with PBS 1\% FCS and stained with FITC conjugated anti-class I and PEconjugated anti-class II monoclonal antibodies or with appropriate isotype controls (PharMingen) for $25 \mathrm{~min}$ on ice, and fixed overnight with $4 \%$ paraformaldehyde. In two experiments, intracellular HLA-DR was detected by treating infected and non-infected fibroblasts cultured with $200 \mathrm{ng} / \mathrm{ml}$ of IFN- $\gamma$ with the BD Cytofix/Cytoperm $^{\mathrm{TM}}$ kit for intracellular staining (BD-Pharmingen) before the addition of anti-class II monoclonal antibodies and isotype control antibody. Stained cells were finally washed and analyzed by flow cytometry using a FACScanto flow cytometer (Becton Dickinson Biosciences, San Jose, CA). From each tube, $2 \times 10^{4}$ viable cells were acquired. Data files were analyzed with the BDFACS Diva software.

\section{8. $T$ cell clones}

The T cell clone RNppd64 specific for purified protein derivative (PPD) and the clone RNF2 specific for the Candida albicans antigen MP65 and for the peptide T1b, an MP65 derived peptide (Tana Laboratories, Houston, Texas) were isolated as previously described [25]. The clones were restimulated with PHA (HA-16 from Murex Diagnostics) in the presence of recombinant human IL-2 (R\&D) and irradiated peripheral blood mononuclear cells as feeder for at least 20-25 days, then cells were washed twice to remove residual IL-2, counted and resuspended in CM.

\subsection{Antigen presentation assays}

To test the capacity of fibroblasts to function as non-professional APCs, the autologous primary human fibroblasts line RNFib was seeded in 96-well flat-bottomed culture plates at $1 \times 10^{4}$ cell/well and allowed to adhere. Cells were then infected with Mtb or BCG (MOI of 3:1 or 10:1, respectively) for $18 \mathrm{~h}$ before the addition of IFN- $\gamma(200 \mathrm{ng} / \mathrm{ml})$. After $72 \mathrm{~h}$, fibroblasts were washed with warm RPMI to remove IFN- $\gamma$ and warm CM was added together with MP65 (a kind gift from Dr. R. La Valle) at $10 \mu \mathrm{g} / \mathrm{ml}$ or T1b at $1 \mu \mathrm{g} / \mathrm{ml}$ and the MP65-specific T cell clone isolated from the same donor of fibroblasts at $3 \times 10^{4} \mathrm{cell} /$ well. After $48 \mathrm{~h}$ activation was measured by IFN- $\gamma$ release in supernatants using an ELISA kit (R\&D Systems) according to the manufacturer's instructions. In a series of experiments, IFN- $\gamma$-treated RNFib were infected overnight with Mtb at different MOI (starting from 30:1), treated with HK Mtb or incubated with Mtb that has been cultured o/n with moxifloxacin $(4 \mu \mathrm{g} / \mathrm{ml})$, kanamycin $(100 \mu \mathrm{g} / \mathrm{ml})$ or isoniazid
( $2 \mu \mathrm{g} / \mathrm{ml})$ or with $5 \mu \mathrm{g} / \mathrm{ml}$ PPD. Then, cells were washed and cocultured with an autologous PPD-specific T cell clone. For some experiments IFN $-\gamma$ treated RNFib were infected o/n with BCG or treated with HK BCG (MOI 10:1) before the addition of autologous responsive $\mathrm{T}$ cell clone.

\subsection{Statistical analysis}

All the statistical analysis were performed using the fourth version of GraphPad Prism Software. The data were analyzed using the non-parametric Mann-Whitney $U$ test. All tests for statistical significance were two-tailed and $p$ values $<0.05$ were considered significant.

\section{Results}

\subsection{Mycobacteria infect human fibroblasts}

The human fibroblasts cell line MRC- 5 and RNFib were infected with Mtb or BCG at MOI of 3:1 or 10:1 respectively. After 2 days of incubation cells were fixed and stained by Kinyoun method and microscopically observed to evaluate the cell-mycobacteria interactions. Fig. 1 reports Kinyoun staining of MRC-5 fibroblasts cultures after BCG (Fig. 1A) or Mtb (Fig. 1B) infection. Mycobacteria are associated to fibroblasts and only rare mycobacterial cells are found in the intercellular spaces. In particular, mycobacteria appear within the fibroblasts, since they often draw the contour of single cells and are observed within fibroblast vacuoli. To confirm the intracellular localization of Mtb in infected fibroblasts, we examined auramine stained mycobacteria after infection by confocal microscopy. The Z-scan of the cell cytoplasm reveals the intracellular localization of mycobacteria soon after a $3 \mathrm{~h}$ infection time (Fig. 1C). Moreover, Mtb or BCG growth in infected human fibroblasts was measured by counting the CFU after 1,3 and 6 days of culture. Mtb or BCG were allowed to infect adherent fibroblasts overnight and then non internalized mycobacteria were removed by vigorous washings. Growth was measured at the time intervals indicated in Fig. 2A (Mtb) and Fig. 2B (BCG) by counting CFU both in supernatants and in lysed fibroblasts as specified in the method section. Starting from day 1, Mtb and BCG begin to replicate within fibroblasts, while only a limited number of CFU are observed in fibroblast free supernatants. Similar results were obtained using RNFib at the same time intervals (data not shown).

\subsection{Mycobacteria reduce the IFN- $\gamma$ mediated up-regulation of $M H C$ class II membrane expression in human fibroblasts}

MHC class II expression on fibroblasts increases almost linearly in the IFN- $\gamma$ range of $12.5-400 \mathrm{ng} / \mathrm{ml}$ (Fig. 3A). Umetsu et al. [22] showed the capacity of fibroblasts to present tetanus toxoid to $\mathrm{CD}^{+} \mathrm{T}$ cell clones by inducing MHC expression on fibroblast with IFN- $\gamma$ at $100 \mathrm{ng} / \mathrm{ml}$. To test the influence of Mtb as well as BCG in the expression and IFN- $\gamma$ mediated induction of MHC class II molecules, uninfected or mycobacteria infected fibroblasts were treated or not with a high dose of recombinant IFN- $\gamma(200 \mathrm{ng} / \mathrm{ml})$ and then cultured for three days before analysis. As shown in Fig. 3B, fibroblasts express MHC class I, but not class II molecules in the absence of stimuli. Infection with both BCG and Mtb causes a slight increase of MHC class I but not class II expression in fibroblasts. Upon IFN- $\gamma$ stimulation of fibroblasts, class I molecule expression is highly up-regulated and expression of class II molecules is induced. However, IFN- $\gamma$ stimulation of Mtb or BCG infected fibroblasts caused a marked reduction of MHC class II molecule expression and a reduced, but not statistically significant, MHC class I up-regulation (Fig. 3B and Table 1). Interestingly, the 

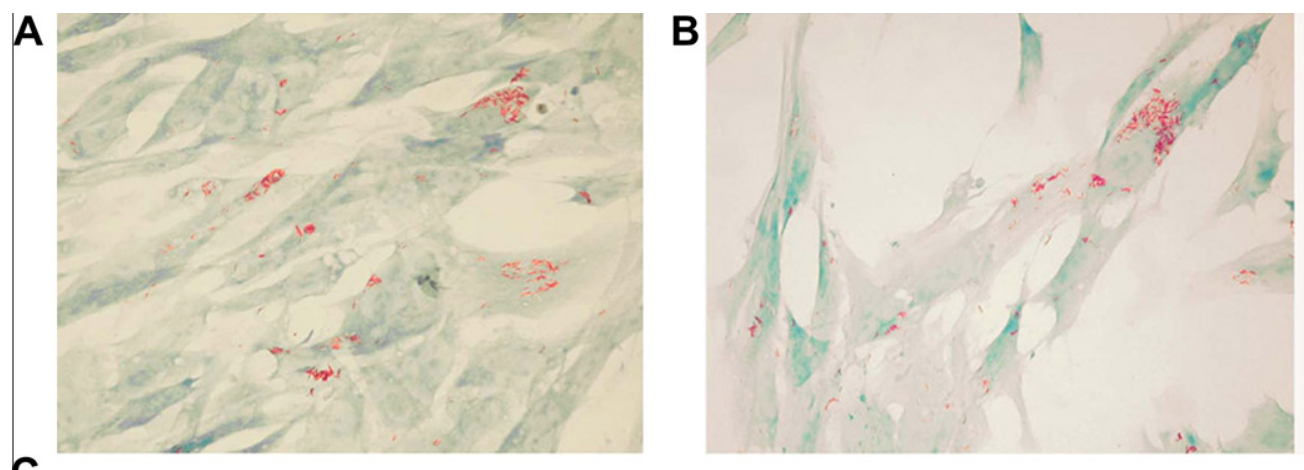

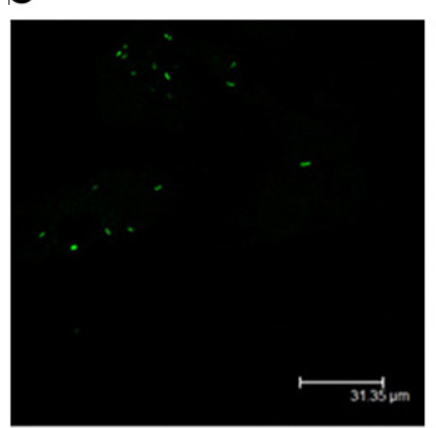

Fluorescence

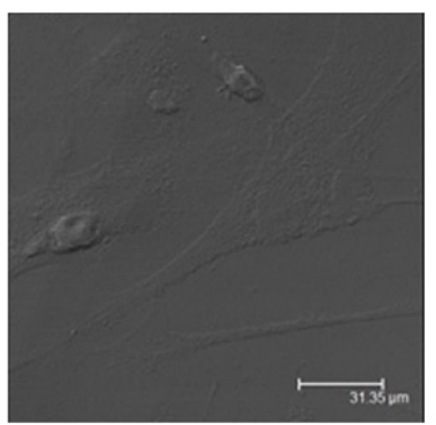

Transmittance

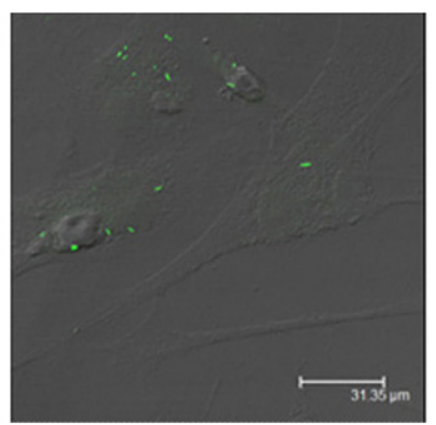

Merge

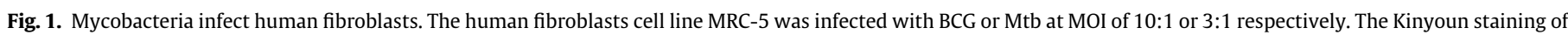

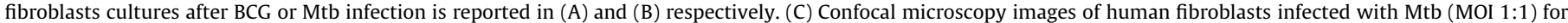

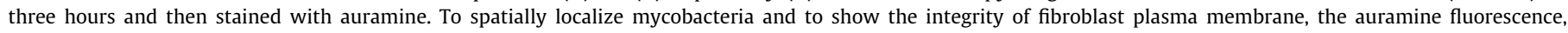
collected from the intracellular microscope focal plane, has been merged with the transmission image by using the Leica TCS-SP2 operating system.
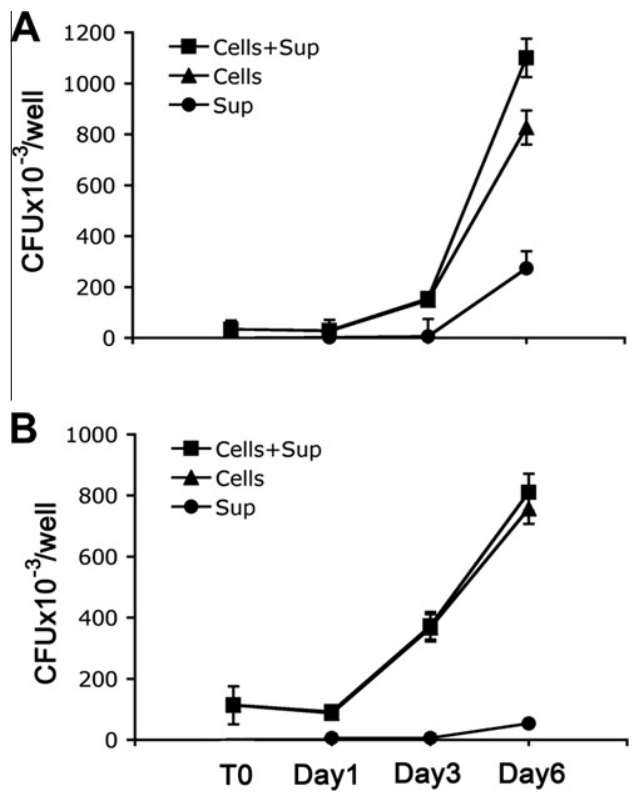

Fig. 2. Mycobacteria grow into human fibroblasts. Mtb (A) or BCG (B) growth in MRC- 5 fibroblasts was followed by counting the CFU after 1,3 and 6 days of culture in comparison to the initial infection dose (T0). The CFU reported refer to numbers of mycobacteria counted in the culture supernatants (Sup), in the fibroblast lysates (cells) and summing the counts in supernatants and cell lysates (Cells + Sup). Values are expressed as a mean of three independent experiments \pm standard deviation. Similar results were obtained using primary human fibroblasts.

MHC class II molecule expression measured after fibroblast permeabilization was not influenced by Mtb infection. In fact, the total amount of MHC class II molecules was found unchanged when both intracellular and cell surface expression were measured (Fig. 3C) suggesting that Mtb does not inhibit MHC class II gene expression, but rather its export to fibroblast cell membrane. To confirm that Mtb infection does not inhibit MHC molecule gene expression, we monitored mRNA changes in response to IFN- $\gamma$ and Mtb infection, by qRT-PCR. We found that HLA class I mRNA was upregulated by Mtb and IFN- $\gamma$, with scarce synergistic effect in combined conditions. Differently, IFN- $\gamma$ modulated the expression of HLA-DR, but we did not observe significant changes in the presence of intracellular Mtb, despite increased expression of MHC class II transcriptional transactivator (CIITA) in IFN- $\gamma$-treated Mtb-infected cells, compared to IFN- $\gamma$ treated non-infected cells (Fig. 4).

\subsection{Mycobacteria restrain the MHC class II dependent antigen presentation by human fibroblasts}

The antigen presenting function of mycobacteria infected fibroblasts was studied in an autologous model. We first show that autologous fibroblasts RNFib are capable of presenting peptides to a specific $\mathrm{CD}^{+} \mathrm{T}$ cell clone only if previously treated with IFN- $\gamma$, that induces the up-regulation of MHC class II (Fig. 5A). Interestingly, Mtb infected fibroblasts are less efficient than uninfected fibroblasts to present the peptide to the $\mathrm{T}$ cell clone. This reduced stimulation of the $T$ cell clone when Mtb infected fibroblasts are used as APCs can be explained by the reduced MHC class II molecule expression observed by immunofluorescence, since peptides do not require processing. To study the possible interference of Mtb in the processing of antigens in nonprofessional APC, infected fibroblasts stimulated with IFN- $\gamma$ were incubated with MP65, a C. albicans mannoprotein and co-cultured with a specific $\mathrm{T}$ cell clone isolated from the same donor of fibroblasts. Fig. 5B shows that fibroblasts are able to process and 

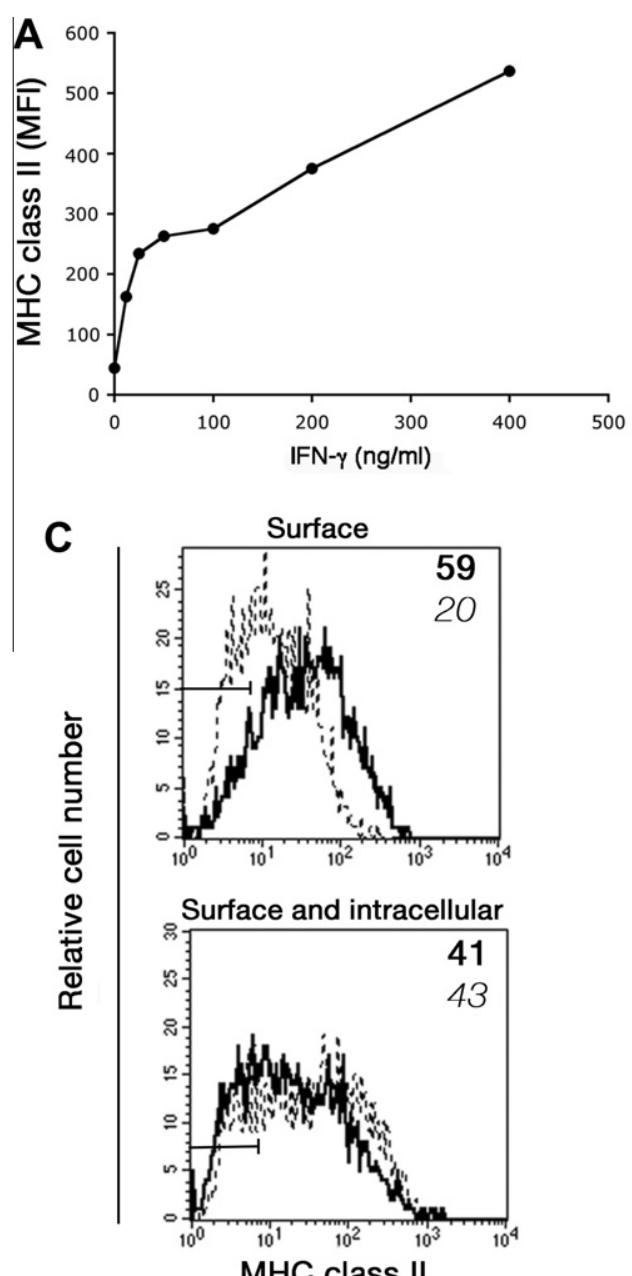

B None
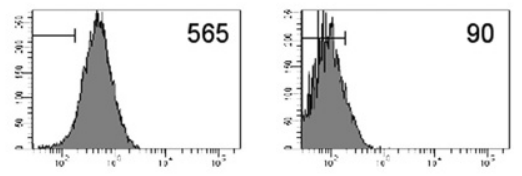

IFN- $\gamma$
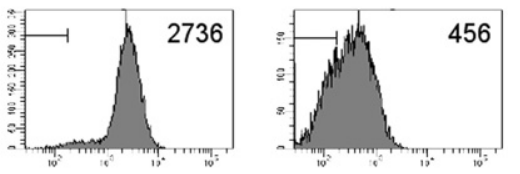

Mtb
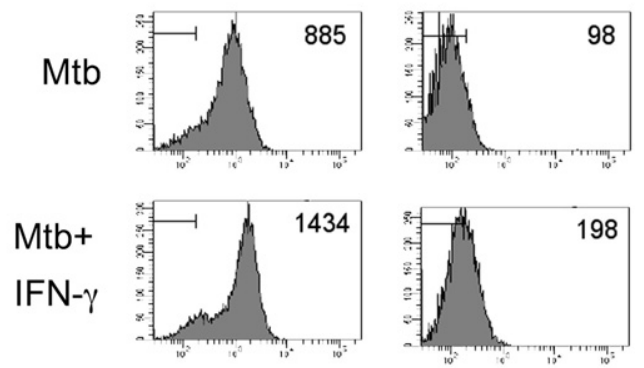

BCG
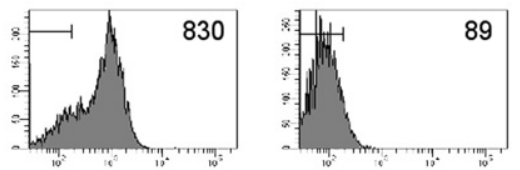

$\mathrm{BCG}+$

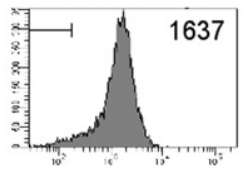

Class I

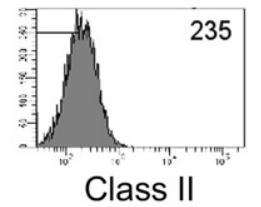

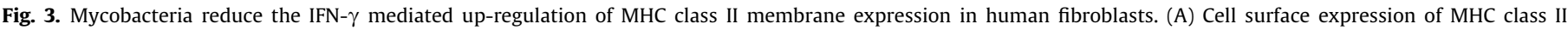

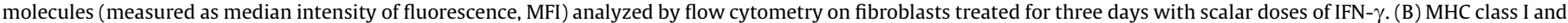

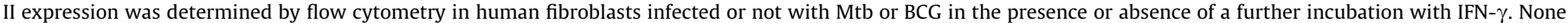

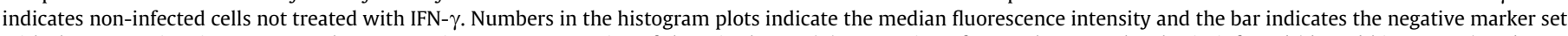

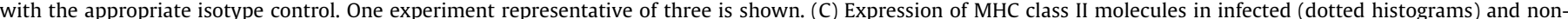

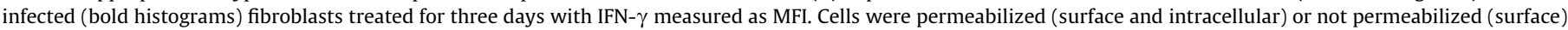

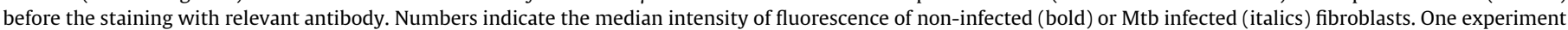
representative of two is shown.

Table 1

Mean reduction of MHC molecule surface expression in infected fibroblasts after treatment with IFN- $\gamma$.

\begin{tabular}{ccc}
\hline & BCG $+\operatorname{IFN}-\gamma\left(n=5^{\mathrm{a}}\right)$ & Mtb $+\operatorname{IFN}-\gamma(n=3)$ \\
\hline MHC class I & $30.7 \pm 26^{\mathrm{b}}$ & $34.3 \pm 22$ \\
& $p=0.83$ & $p=0.18$ \\
MHC class II & $46.4 \pm 7.9$ & $57.8 \pm 9.5$ \\
& $p<0.01^{*}$ & $p<0.05^{*}$
\end{tabular}

${ }^{\mathrm{a}} n=$ number of independent experiments.

b Percentage MFI reduction in comparison to non-infected IFN- $\gamma$ treated fibroblasts \pm SD.

$p$ Values statistically significant.

present the soluble protein MP65 to the specific T cell clone only if treated with the MHC class II inducer IFN- $\gamma$. More interestingly, Mtb, and to a lesser extent BCG, block the presentation capacity of fibroblasts to the T cell clone, which in turns is not stimulated to secrete IFN- $\gamma$.

Moreover, to test the capacity of fibroblasts to process Mtb and to present its specific antigens, IFN- $\gamma$ stimulated fibroblasts were infected with viable Mtb (MOI 3:1) or treated with Mtb killed by heat or by o/n culture with moxifloxacin, kanamycin or isoniazid. As a read out, the IFN- $\gamma$ release by an autologous PPD specific T cell clone was used. Data show that IFN- $\gamma$ stimulated fibroblasts have the capacity to present antigens derived from the processing of a whole bacterium, since they are able to act as APCs for the HK Mtb (Fig. 5C). However, when infected with the viable Mtb, fibroblasts are unable to process and present Mtb derived antigens, suggesting that viable mycobacteria actively interfere with the presentation and processing of fibroblasts. Interestingly, not only HK, but also moxifloxacin, kanamycin and isoniazid treated Mtb (Fig. 5D) were also processed by fibroblasts and presented to specific $\mathrm{T}$ cell clones.

\section{Discussion}

In this work we confirm that in vitro Mtb infects human fibroblasts. Infection seems not to be related to the virulence of Mtb, since also the vaccine strain BCG is endowed with the same capacity to infect and grow within fibroblasts. These observations are in line with previous published data demonstrating the capacity of Mtb, as well as attenuated or non-pathogenic mycobacteria such 


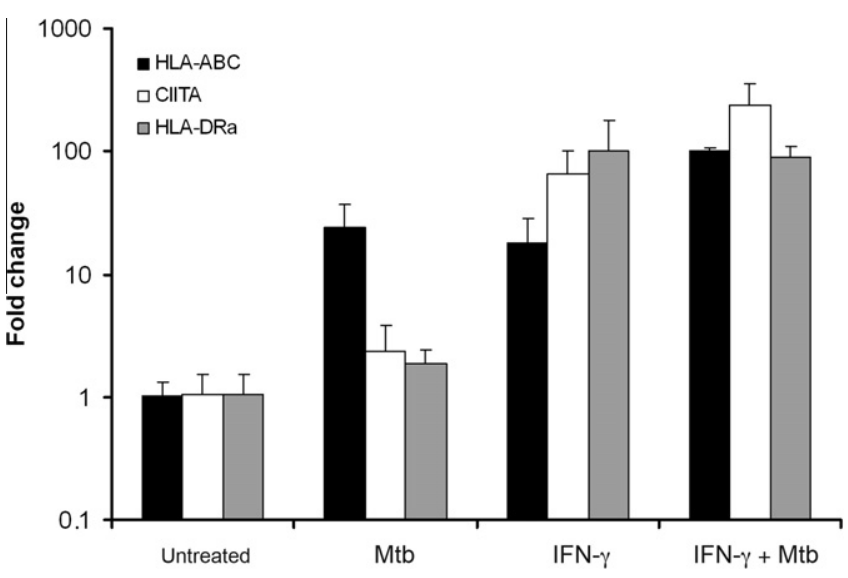

Fig. 4. MHC gene expression in Mtb-infected and IFN- $\gamma$-treated fibroblasts is not reduced. RNA from control fibroblasts, Mtb infected, IFN- $\gamma$ treated and the two last conditions combined were tested by qRT-PCR with primers specific for common regions of HLA-A, -B and -C genes, for transcription factor CIITA and HLA-DR $\alpha$ genes. Expression was quantified as fold changes compared to control cells, using Betaactin as housekeeping gene, calculated using the $2^{-\Delta \Delta \mathrm{Ct}}$ formula. Data $\pm \mathrm{SD}$ are from three independent infection experiments.

as $M$. smegmatis, to infect non-phagocytic cells [8-11] by macropinocytosis through lamellipodia formation and cell cytoskeleton rearrangement $[4,13]$.

Since the intracellular growth of mycobacteria in phagocytes may be limited by the intervention of the adaptive immune system, namely by the secretion of inflammatory cytokines following the recognition of specific antigens by effector $\mathrm{T}$ cells, Mtb has evolved an escape strategy based on the inhibition of phagosome maturation [2] and the modulation of MHC class II molecules $[3,26]$. Previous studies have so far analyzed the interference of mycobacteria with MHC class II expression in professional phagocytes, such as macrophages or DCs. These cells are characterized by a constitutional expression of MHC class II molecules that may be increased by the release of cytokines such as IFN- $\gamma$ or by inflammatory stimuli and bacterial products [27]. Here we investigate whether mycobacteria infection also interferes with de novo expression of MHC class II molecules in non-phagocytic cells upon IFN- $\gamma$ stimulation using fibroblasts as a prototype. In particular, we focus on fibroblasts since they are crucial players in granuloma formation [28] and they were previously found to host Mtb in LTBI [17].

We demonstrate that fibroblasts infected by mycobacteria upregulate MHC class II molecules to a statistically significant lower extent than non-infected cells upon IFN $-\gamma$ stimulation. This effect is observed in vitro even at very high IFN- $\gamma$ doses (up to $400 \mathrm{ng}$ / $\mathrm{ml}$ ) so that the inhibition of MHC class II molecule expression induced by mycobacteria is likely to occur also in vivo, where IFN- $\gamma$ levels may be lower that those used in vitro [29]. Interestingly, MHC class I molecules were up-regulated following infection with Mtb or BCG, in terms of both mRNA levels and membrane expression. IFN- $\gamma$ treatment of fibroblasts caused an increase of MHC class I mRNA synthesis and membrane expression. MHC class I mRNA levels were found only weakly increased upon IFN- $\gamma$ treatment of Mtb infected fibroblasts, suggesting that IFN- $\gamma$ and Mtb are not additive stimuli for MHC class I mRNA synthesis. However, MHC class I membrane expression in infected and IFN- $\gamma$ treated fibroblasts increased, but to a lesser and not statistically significant extent in comparison to non-infected fibroblasts. These data suggest that Mtb infection causes a reduced MHC class I membrane export of preformed molecules in IFN- $\gamma$ treated fibroblasts. These findings are in agreement with previous works showing that Mtb is able to interfere with MHC class II but not class I molecules
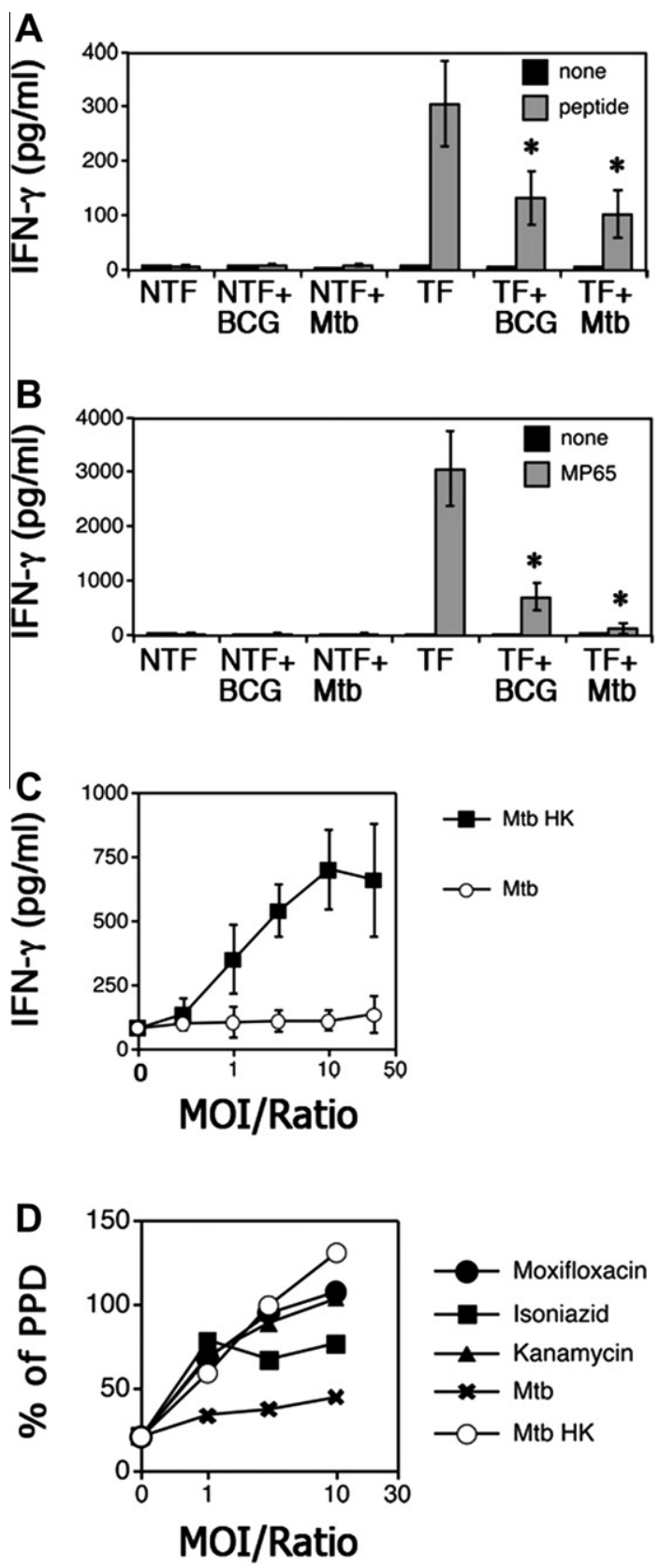

Fig. 5. Mycobacteria interfere with the MHC class II dependent antigen presentation by human fibroblasts. MHC class II dependent response of a MP65 specific T cell clone to a peptide of MP65 (A) or to the MP65 soluble mannoprotein (B) in the presence of autologous fibroblasts treated (TF) or non treated (NTF) with IFN- $\gamma$ after the infection with Mtb, BCG or left uninfected. The $\mathrm{T}$ cell clone activation was measured as IFN $-\gamma$ release and expressed as $\mathrm{pg} / \mathrm{ml} \pm \mathrm{SD}$ of three independent experiments. The asterisk indicates a significant difference $(p<0.05)$ between infected and non-infected TF. (C) IFN- $\gamma$ pre-treated fibroblasts were infected with viable (Mtb) or incubated with heat-killed (HK) Mtb at different MOI or ratios, respectively, then washed and co-cultured with autologous PPD-specific T cell clone $\mathrm{T}$ cell activation was measured as IFN $-\gamma$ release and expressed as $\mathrm{pg} / \mathrm{ml} \pm$ standard deviation of three independent experiments. (D) IFN- $\gamma$-treated fibroblasts were incubated o/n with PPD or with viable or heat killed (HK) Mtb or with Mtb that has been pre-cultured o/n with moxifloxacin $(4 \mu \mathrm{g} / \mathrm{ml})$, kanamycin $(100 \mu \mathrm{g} / \mathrm{ml})$ or isoniazid $(2 \mu \mathrm{g} / \mathrm{ml})$, then washed and co-cultured with an autologous PPD-specific T cell clone. T cell activation was measured as IFN- $\gamma$ release and expressed as percentage of IFN $-\gamma$ production respect to the activation obtained with PPD. One experiment representative of two is shown.

expression on macrophages [26,30], and may be linked to the constitutive expression of MHC class I molecules on both professional and non-professional APCs. 
Mtb uses diverse strategies to interfere with MHC class II expression in macrophages [31], including the reduction of the MHC class II transcriptional transactivator (CIITA) activity, thus causing the reduced transcription of MHC class II mRNA [32,33]. Moreover, Mtb can interfere with the processing of the MHC class II invariant chain reducing the MHC class II export to cell membrane through the inhibition of phagosomal maturation and activity of proteolytic enzymes such as cathepsines [34,35]. Our results indicate that the observed reduced MHC class II membrane expression is not dependent on the Mtb inhibition of CIITA and MHC class II transcription. Although the aim of this study was not the identification of the mechanisms causing the observed phenomenon, our data suggest that in fibroblasts Mtb inhibits the MHC class II membrane expression by interfering with the intracellular transport of MHC class II to the external membrane, possibly inhibiting the activity of enzymes involved in the processing of the invariant chain directly or indirectly by modifying the acidity of the Mtb containing vacuoli.

The term non-professional APCs is related to cells on which expression of MHC class II molecules can be induced by IFN- $\gamma$, such as endothelial cells [36], myoblasts [37], or fibroblasts [22]. The antigen presentation by non-professional APCs can support proliferation of T cell clones or memory T cells, i.e., when co-stimulation is not required. On the contrary, they are unable to initiate a primary response by naive $T$ lymphocytes. Accordingly, the capacity to present soluble antigen by fibroblasts has probably scarce significance in T cell priming, since professional APCs in primary lymphoid organs are the cells deputed to this function. However, the capacity of fibroblasts to present antigens during recall immune responses may have relevance in the fight against pathogens. In fact, non-professional APCs, outnumbering professional APCs in tissues, may contribute to modulate the magnitude of the efferent phase of an immune response by activating memory T lymphocytes.

To test the functional consequence of MHC class II reduced induction in non-professional APCs, we first used the capacity of infected fibroblasts to present peptides in an autologous model. We used a peptide synthesized based on the epitope sequence of MP65, an immunodominant antigen of $C$. albicans previously shown to cause the activation of our $T$ cell clones. Since peptides do not require internalization and processing by APC, the activation of a specific $\mathrm{T}$ cell clone can be considered as the functional expression of MHC class II expression on fibroblasts upon IFN- $\gamma$ treatment [38]. We observed that the peptide presentation is reduced but not completely abrogated in mycobacteria infected fibroblasts. This result is in agreement with the reduced but not completely inhibited MHC class II membrane expression observed in infected fibroblasts. Moreover, we tested the capacity of mycobacteria to interfere with the antigen presentation of a protein antigen by non-professional APCs. To compare the APCs function of Mtb infected fibroblasts to that of non-infected cells, we used the protein MP65, an antigen that is not shared by Mtb [25], and a MP65 specific T cell clone activation as a readout. The Mtb infection causes an almost complete inhibition of the presentation of MP65 to the specific T cell clone by fibroblasts even if the MHC class II expression is reduced but not abrogated. Moreover, using a PPD specific $\mathrm{T}$ cell clone, we demonstrate an almost complete inability of Mtb infected fibroblasts to present mycobacterial antigens, indicating that Mtb, by infecting fibroblasts, hides itself to the recognition of $\mathrm{CD}^{+} \mathrm{T}$ lymphocytes. The failure of fibroblasts to present Mtb antigens is not dependent on their intrinsic inability to process whole bacteria. In fact, fibroblasts induced the activation of the PPD specific clone if treated with Mtb killed by heat or treated with specific antibiotics. This result indicates that fibroblasts are cells endowed with the capacity to process whole bacteria in an environment characterized by the presence of IFN- $\gamma$, expanding our understanding of the non-professional APC function of fibroblasts [22]. Lastly, our data suggest that the capacity of Mtb to restrain the APC function of fibroblasts is an active phenomenon requiring metabolically active and viable Mtb cells. Thus, the interference with the fibroblast antigen presentation capacity seems not to be dependent on structural components of the bacterium.

It can be hypothesized that fibrotic TB lesions with low oxygen concentrations represent a hostile microenvironment that may favor the Mtb transition to the non-replicating status of dormancy. Since our data indicate that in fibroblasts Mtb may also escape helper $\mathrm{T}$ cell recognition, it is possible to speculate that fibroblasts may represent a Mtb reservoir in LTBI. In this line, it is interesting to note that Mtb has been described in fibroblasts in LTBI [17], but not in active TB. Considering that in vitro both human and mouse [39] fibroblasts are permissive to Mtb growth, it is not clear why fibroblasts, that are associated with tuberculous loci and participate in granuloma formation have not been found to contain Mtb in vivo. A possible explanation is that in active diseases the growth in macrophages may be favored and infection of fibroblasts or other cells is sporadic and thus difficult to detect. The other possibility is that fibroblast may represent an immunoprivileged site for Mtb where to persist in the latent infection only. In conclusion, we have confirmed that Mtb can infect human fibroblasts [13,14] and propose a possible functional consequence of the fibroblast infection in terms of escape from the host immune response. Even if in vitro data cannot completely reproduce phenomena occurring in vivo, we report data that increase our knowledge on the possible mechanisms used by Mtb to persist in immunocompetent individuals. In fact, if infected in vivo, fibroblasts may represent immunoprivileged cells for Mtb homing and it can be speculated that, hidden to the helper $\mathrm{T}$ cell recognition, Mtb may grow in fibroblasts during active TB or to persist in a dormant status during latent TB infection.

\section{Acknowledgments}

This work was partially supported by the 7th EC Framework Programme Project “NEWTBVac” Grant \#241745.

\section{References}

[1] Kaufmann SH, Schaible UE. A dangerous liaison between two major killers: Mycobacterium tuberculosis and HIV target dendritic cells through DC-SIGN. J Exp Med 2003;197:1.

[2] Brodin P, Poquet Y, Levillain F, Peguillet I, Larrouy-Maumus G, Gilleron M, et al. High content phenotypic cell-based visual screen identifies Mycobacterium tuberculosis acyltrehalose-containing glycolipids involved in phagosome remodeling. PLoS Pathog 2010;6:e1001100.

[3] Harding CV, Boom WH. Regulation of antigen presentation by Mycobacterium tuberculosis: a role for Toll-like receptors. Nat Rev Microbiol 2010;8:296.

[4] Garcia-Perez BE, Hernandez-Gonzalez JC, Garcia-Nieto S, Luna-Herrera J. Internalization of a non-pathogenic mycobacteria by macropinocytosis in human alveolar epithelial A549 cells. Microb Pathog 2008;45:1.

[5] Sato K, Tomioka H, Shimizu T, Gonda T, Ota F, Sano C. Type II alveolar cells play roles in macrophage-mediated host innate resistance to pulmonary mycobacterial infections by producing proinflammatory cytokines. J Infect Dis 2002;185:1139.

[6] Shepard CC. Use of HeLa cells infected with tubercle bacilli for the study of antituberculous drugs. J Bacteriol 1957;73:494.

[7] Shepard CC. A study of the growth in HeLa cells of tubercle bacilli from human sputum. Am Rev Tuberc 1958;77:423.

[8] Bermudez LE, Sangari FJ, Kolonoski P, Petrofsky M, Goodman J. The efficiency of the translocation of Mycobacterium tuberculosis across a bilayer of epithelial and endothelial cells as a model of the alveolar wall is a consequence of transport within mononuclear phagocytes and invasion of alveolar epithelial cells. Infect Immun 2002;70:140.

[9] Bermudez LE, Goodman J. Mycobacterium tuberculosis invades and replicates within type II alveolar cells. Infect Immun 1996;64:1400.

[10] Mehta PK, King CH, White EH, Murtagh Jr JJ, Quinn FD. Comparison of in vitro models for the study of Mycobacterium tuberculosis invasion and intracellular replication. Infect Immun 1996;64:2673.

[11] Bermudez LE, Shelton K, Young LS. Comparison of the ability of Mycobacterium avium, $M$. smegmatis, and $M$. tuberculosis to invade and replicate within HEp-2 epithelial cells. Tuberc Lung Dis 1995;76:240. 
[12] Neyrolles O, Hernandez-Pando R, Pietri-Rouxel F, Fornes P, Tailleux L, Barrios Payan JA, et al. Is adipose tissue a place for Mycobacterium tuberculosis persistence? PLoS One 2006;1:e43.

[13] Ferrer NL, Gomez AB, Neyrolles O, Gicquel B, Martin C. Interactions of attenuated Mycobacterium tuberculosis phoP mutant with human macrophages. PLoS One 2010;5:e12978.

[14] Byrd TF, Green GM, Fowlston SE, Lyons CR. Differential growth characteristics and streptomycin susceptibility of virulent and avirulent Mycobacterium tuberculosis strains in a novel fibroblast-mycobacterium microcolony assay. Infect Immun 1998;66:5132.

[15] Tailleux L, Neyrolles O, Honore-Bouakline S, Perret E, Sanchez F, Abastado JP, et al. Constrained intracellular survival of Mycobacterium tuberculosis in human dendritic cells. J Immunol 2003;170:1939.

[16] Wolf AJ, Linas B, Trevejo-Nunez GJ, Kincaid E, Tamura T, Takatsu K, et al. Mycobacterium tuberculosis infects dendritic cells with high frequency and impairs their function in vivo. J Immunol 2007;179:2509.

[17] Hernandez-Pando R, Jeyanathan M, Mengistu G, Aguilar D, Orozco H, Harboe $\mathrm{M}$, et al. Persistence of DNA from Mycobacterium tuberculosis in superficially normal lung tissue during latent infection. Lancet 2000;356:2133.

[18] Haque A, Hajiaghamohseni LM, Li P, Toomy K, Blum JS. Invariant chain modulates HLA class II protein recycling and peptide presentation in nonprofessional antigen presenting cells. Cell Immunol 2007;249:20.

[19] Debbabi H, Ghosh S, Kamath AB, Alt J, Demello DE, Dunsmore S, et al. Primary type II alveolar epithelial cells present microbial antigens to antigen-specific CD4+ T cells. Am J Physiol Lung Cell Mol Physiol 2005;289:L274.

[20] Bentley AG, Phillips SM, Kaner RJ, Theodorides VJ, Linette GP, Doughty BL. In vitro delayed hypersensitivity granuloma formation: development of an antigen-coated bead model. J Immunol 1985;134:4163.

[21] Mariotti S, Teloni R, Iona E, Fattorini L, Giannoni F, Romagnoli G, et al. Mycobacterium tuberculosis subverts the differentiation of human monocyte into dendritic cell. Eur J Immunol 2002;32:3050.

[22] Umetsu DT, Pober JS, Jabara HH, Fiers W, Yunis EJ, Burakoff SJ, et al. Human dermal fibroblasts present tetanus toxoid antigen to antigen-specific $\mathrm{T}$ cell clones. J Clin Invest 1985;76:254.

[23] Pattyn F, Speleman F, De Paepe A, Vandesompele J. RTPrimerDB: the real-time PCR primer and probe database. Nucleic Acids Res 2003;31:122.

[24] Greco E, Santucci MB, Sali M, De Angelis FR, Papi M, De Spirito M, et al. Natural lysophospholipids reduce Mycobacterium tuberculosis-induced cytotoxicity and induce anti-mycobacterial activity by a phagolysosome maturationdependent mechanism in A549 type II alveolar epithelial cells. Immunology 2010;129:125.

[25] Nisini R, Romagnoli G, Gomez MJ, La Valle R, Torosantucci A, Mariotti S, et al. Antigenic properties and processing requirements of 65-kilodalton mannoprotein, a major antigen target of anti-Candida human T-cell response, as disclosed by specific human $\mathrm{T}$-cell clones. Infect Immun 2001;69:3728.
[26] Pai RK, Convery M, Hamilton TA, Boom WH, Harding CV. Inhibition of IFNgamma-induced class II transactivator expression by a 19-kDa lipoprotein from Mycobacterium tuberculosis: a potential mechanism for immune evasion. J Immunol 2003;171:175.

[27] Cella M, Engering A, Pinet V, Pieters J, Lanzavecchia A. Inflammatory stimuli induce accumulation of MHC class II complexes on dendritic cells. Nature 1997;388:782.

[28] Barry 3rd CE, Boshoff HI, Dartois V, Dick T, Ehrt S, Flynn J, et al. The spectrum of latent tuberculosis: rethinking the biology and intervention strategies. Nat Rev Microbiol 2009; 7:845.

[29] Rai AK, Thakur CP, Singh A, Seth T, Srivastava SK, Singh P, et al. Regulatory T cells suppress $\mathrm{T}$ cell activation at the pathologic site of human visceral leishmaniasis. PLoS One 2012;7:e31551.

[30] Noss EH, Pai RK, Sellati TJ, Radolf JD, Belisle J, Golenbock DT, et al. Toll-like receptor 2-dependent inhibition of macrophage class II MHC expression and antigen processing by $19-\mathrm{kDa}$ lipoprotein of Mycobacterium tuberculosis. J Immunol 2001;167:910.

[31] Baena A, Porcelli SA. Evasion and subversion of antigen presentation by Mycobacterium tuberculosis. Tissue Antigens 2009;74:189.

[32] Fulton SA, Reba SM, Pai RK, Pennini M, Torres M, Harding CV, et al. Inhibition of major histocompatibility complex II expression and antigen processing in murine alveolar macrophages by Mycobacterium bovis BCG and the 19kilodalton mycobacterial lipoprotein. Infect Immun 2004;72:2101.

[33] Pennini ME, Pai RK, Schultz DC, Boom WH, Harding CV. Mycobacterium tuberculosis 19-kDa lipoprotein inhibits IFN-gamma-induced chromatin remodeling of MHC2TA by TLR2 and MAPK signaling. J Immunol 2006;176:4323.

[34] Hestvik AL, Hmama Z, Av-Gay Y. Mycobacterial manipulation of the host cell. FEMS Microbiol Rev 2005;29:1041.

[35] Sendide K, Deghmane AE, Pechkovsky D, Av-Gay Y, Talal A, Hmama Z Mycobacterium bovis BCG attenuates surface expression of mature class II molecules through IL-10-dependent inhibition of cathepsin S. J Immunol 2005; $175: 5324$.

[36] Marelli-Berg FM, Hargreaves RE, Carmichael P, Dorling A, Lombardi G, Lechler RI. Major histocompatibility complex class II-expressing endothelial cells induce allospecific nonresponsiveness in naive $T$ cells. $J$ Exp Med 1996;183:1603.

[37] Goebels N, Michaelis D, Wekerle H, Hohlfeld R. Human myoblasts as antigenpresenting cells. J Immunol 1992;149:661.

[38] Drozina G, Kohoutek J, Jabrane-Ferrat N, Peterlin BM. Expression of MHC II genes. Curr Top Microbiol Immunol 2005;290:147.

[39] Rastogi N, Labrousse V, de Sousa JP. Mycobacterial growth and ultrastructure in mouse L-929 fibroblasts and bone marrow-derived macrophages: evidence that infected fibroblasts secrete mediators capable of modulating bacterial growth in macrophages. Curr Microbiol 1992;25:203. 